(C) 2000 International Press

Adv. Theor. Math. Phys. 3 (2000) 567-576

\title{
An obstructed bundle on a
}

\section{Calabi-Yau 3-fold}

\author{
R.P. Thomas
}

\begin{abstract}
Mirror symmetry suggests that on a Calabi-Yau 3-fold moduli spaces of stable bundles, especially those with degree zero and indivisible Chern class, might be smooth (i.e., unobstructed, though perhaps of too high a dimension). This is because smoothly embedded special lagrangian cycles in the mirror have unobstructed deformations. As there does not seem to be a counterexample in the literature we provide one here, showing that such a Tian-Todorov-McLean-type result cannot hold.
\end{abstract}

\section{Introduction}

The existence of curves $C$ in Calabi-Yau 3-folds $X$ whose deformations are obstructed has been known for some time. This means that the curve deforms to first order - the linearisation of the deformation problem has an $H^{0}(\nu)$ of solutions (where $\nu$ is the normal bundle to $C \subset X$ ) - but the actual deformation problem has no solutions (in some direction in $H^{0}(\nu)$ at least). Thus the moduli space of curves is not smooth at this point but has nilpotent directions.

e-print archive: http://xxx.lanl.gov/math.AG/9903034 
This implies that moduli spaces of sheaves can also be obstructed (by taking the ideal sheaves of the curves). However, the now folklore philosophy (see for instance [Ty], [Va]) of the relationship between the Kontsevich and Strominger-Yau-Zaslow approaches to mirror symmetry suggests that for bundles the situation might be different. Namely, under certain circumstances, degree 0 stable (Hermitian-Yang-Mills) bundles on a Calabi-Yau 3-fold $X$ should correspond to special Lagrangian cycles (with flat line bundles on them) on the mirror $\widetilde{X}$. This would be set up by some sort of analytical version of the relative FourierMukai transform for the dual $T^{3}$-fibrations of $X$ and $\widetilde{X}$ whose existence is conjectured by Strominger-Yau-Zaslow. And McLean [Mc] has shown that deformations of smoothly embedded special Lagrangians are unobstructed.

In general the picture is more complicated, with complexes of sheaves on one side corresponding to elements of the derived category of the Fukaya category of all Lagrangians on the other. Sheaves on one side can be obstructed, while on the other singular Lagrangians and "multiply wrapped" cycles (images of degree $>1$ maps, or cycles with higher rank vector bundles on them) may not have smooth moduli. But the question remains as to what the analogue of the McLean's result is in terms of sheaves on $X$. A natural subcategory of the derived category of coherent sheaves on $X$ is the category of holomorphic vector bundles, and while the mirror situation to having multi-wrapped components is hard to understand, it is clearly related to divisibility of the homology classes concerned. Thus it was conjectured that moduli spaces of stable holomorphic bundles of degree zero and indivisible Chern class on Calabi-Yau 3-folds might be smooth. This would be analogous to McLean's result and also the Tian-Todorov theorem that moduli spaces of the Calabi-Yau manifolds themselves are smooth, again despite the fact that they can be of the wrong dimension with a non-zero obstruction space.

Here, however, we will show that this cannot be true. We work on a very particular smooth (3,3)-divisor $X \subset P=\mathbb{P}^{2} \times \mathbb{P}^{2}$ described below. By the Lefschetz hyperplane theorem [GH], $H^{2}(X) \cong H^{2}(P)$ is generated, over the integers, by the standard Kähler forms $\omega_{1}, \omega_{2}$ pulled back from the two $\mathbb{P}^{2}$ factors of $P$. By Poincaré duality $H^{4}(X)$ is generated over the integers by $\omega_{1}^{2} / 3$ and $\omega_{2}^{2} / 3$, with $\omega_{1} \omega_{2}=\omega_{1}^{2}+\omega_{2}^{2}$. 
Theorem 1.1 There exists a rank 2 holomorphic vector bundle $A$ on $X$ such that

- $c_{1}(A)=0, c_{2}(A)=\omega_{1}^{2}+4 / 3 \omega_{2}^{2}$ is indivisible,

- $A$ is slope-stable (and so Gieseker-stable) with respect to the Kähler forms $N \omega_{1}+\omega_{2}, N>1$, and

- deformations $H^{1}($ End $A) \cong \mathbb{C}^{2}$ are completely obstructed to second order.

Thus the component of the moduli space that $A$ sits in is a thickened point, with ring of functions $\mathbb{C}[\epsilon, \eta] /\left(\epsilon^{2}, \epsilon \eta, \eta^{2}\right)$.

The strategy is to employ the Serre construction relating vector bundles to curves via zeros of a section. The work is in finding an obstructed (rational) curve $C$ such that a bundle $A$ can be constructed from it, and such that $A$ is stable (rational curves usually correspond to unstable bundles) with deformation theory exactly that of $C$.

It would be interesting to know what the mirror object to $A$ is. Although $c_{2}(A)$ is indivisible, it is the sum of two divisible classes which are positive in some sense, and so may correspond to some kind of sum of two multiply wrapped cycles, or some more complicated (obstructed) object of the derived category of the Fukaya category of the mirror. Also it is natural to expect the correspondence between stable bundles and special Lagrangians to hold only in the adiabatic limit of small SYZ $T^{3}$-fibres, where it is possible that $A$ is not stable.

\section{Acknowledgements}

I would like to thank Sheldon Katz for pointing out [K] which prompted this work (though its influence is well hidden now), and the organisers of the Harvard Winter School on Mirror Symmetry in January 1999 for the stimulating atmosphere in which the prompting occured. Thanks also to Ivan Smith for useful conversations. 


\section{An obstructed rational curve}

Consider a Calabi-Yau 3-fold $X=X_{3,3} \subset P$, a (3,3) divisor in $P=$ $\mathbb{P}^{2} \times \mathbb{P}^{2}=\mathbb{P}_{1}^{2} \times \mathbb{P}_{2}^{2}$. This is elliptically fibred over the second factor $\mathbb{P}_{2}^{2}$, and we will construct a smooth example with a degenerate fibre $f$ that is the union of a line with a double (thickened) line

$$
f=\left\{x^{2} y=0\right\} \subset \mathbb{P}_{1}^{2} .
$$

Moving away from this fibre we will have either smooth tori fibres, or degenerate fibres that are unions of smooth conics and lines - thus the line $C=\{x=0\}$ whose double lies in $f$ cannot deform inside $X$ beyond its first order deformations inside the thickening.

Fixing local coordinates $(u, v)$ on the base $\mathbb{P}_{2}^{2}$, and coordinates $[x$ : $y: z]$ on $\mathbb{P}_{1}^{2}$, a local example of such a $(3,3)$ divisor is given by the zero locus of

$$
F=\left(x^{2}+z^{2} v\right) y+z^{3} u+(x+y)^{3} v+p,
$$

where the degenerate fibre $f$ is at $u=0=v$, and $p$ is any small perturbation vanishing on $f$. We have

$$
\begin{aligned}
& \left.\frac{\partial F}{\partial u}\right|_{u=0=v}=z^{3}+\frac{\partial p}{\partial u} \\
& \left.\frac{\partial F}{\partial v}\right|_{u=0=v}=z^{2} y+(x+y)^{3}+\frac{\partial p}{\partial v} .
\end{aligned}
$$

Thus for $p$ sufficiently small that $\partial p / \partial u$ is pointwise smaller in norm than $z^{3}$ away from a fixed small neighbourhood of $\{z=0\} \subset \mathbb{P}_{1}^{2}$, and $\partial p / \partial v$ pointwise smaller in norm than $z^{2} y+(x+y)^{3}$ away from a fixed small neighbourhood of $\left\{z^{2} y+(x+y)^{3}=0\right\}$, we have $X=F^{-1}(0)$ smooth in a neighbourhood of $u=0=v: \partial F / \partial u$ and $\partial F / \partial v$ vanish simultaneously only in a small neighbourhood of the intersection of $z=0$ and $z^{2} y+(x+y)^{3}=0$, but this is $[1:-1: 0] \in \mathbb{P}_{1}^{2} \times\{(0,0)\}$ which does not lie on $X$.

Globally then, with respect to coordinates $[x: y: z]$ on $\mathbb{P}_{1}^{2}$ and $[u: v: w]$ on $\mathbb{P}_{2}^{2}$, we set

$$
\begin{aligned}
& F=x^{2} y w^{3}+z^{2} y v w^{2}+z^{3} u w^{2}+(x+y)^{3} v w^{2}+p \quad \in H^{0}(P, \mathcal{O}(3(\text { B.) })) \\
& X=F^{-1}(0) \supset f=\left\{x^{2} y=0=u=v\right\}
\end{aligned}
$$


for any $p$ vanishing on $f$ and sufficiently small (as above) for $F^{-1}(0)$ to be smooth around $f$. The set of such $F$ s form the linear system

$$
H^{0}\left(P, \mathcal{I}_{f}(3,3)\right) \subset H^{0}(P, \mathcal{O}(3,3))
$$

where $\mathcal{I}_{f}(3,3)$ denotes the sheaf of functions on $P$ vanishing on $f$, twisted by the line bundle $\mathcal{O}_{P}(3,3)$. It is easy to see that the base locus of this linear system is just $f \subset P$ : we want to show that for any $x \in P \backslash f$, there exists an element of the linear system not vanishing on $x$. This is clear if $x$ does not lie in the $\mathbb{P}_{1}^{2}$ fibre over $u=0=v$ that contains $f$, and if it does we simply take one of the $F$ s as above.

Thus, by Bertini's theorem [GH], the generic element of the linear system has smooth zero locus away from $u=0=v$. In particular we may chose $p$ arbitrarily small so that $X$ is both smooth on $u=0=v$, as above, and away from it.

We now fix such a $p$, giving an $F$ and a smooth $X$, and study the deformations of the line $C=\{x=0=u=v\} \subset f \subset X$ inside $X$.

Lemma $2.2 \nu_{C / X} \cong \mathcal{O}_{C}(1) \oplus \mathcal{O}_{C}(-3)$, so that $H^{0}\left(\nu_{C / X}\right) \cong \mathbb{C}^{2}$.

Proof. It is clear that $\nu_{C / P}=\nu_{C / \mathbb{P}_{1}^{2}} \oplus \nu_{\mathbb{P}_{1}^{2} / P} \cong \mathcal{O}_{C}(1) \oplus \mathcal{O}_{C} \oplus \mathcal{O}_{C}$, where $\mathbb{P}_{1}^{2}=\mathbb{P}_{1}^{2} \times\{[0: 0: 1]\} \subset \mathbb{P}_{1}^{2} \times \mathbb{P}_{2}^{2}=P$. We set $w=1$ and work with coordinates $[x: y: z], u, v$.

The standard exact sequence

$$
\left.0 \rightarrow \nu_{C / X} \rightarrow \nu_{C / P} \stackrel{d F}{\longrightarrow} \mathcal{O}(3,3)\right|_{C} \rightarrow 0
$$

shows that $\nu_{C / X}$ is the kernel of the map

$$
\mathcal{O}_{C}(1) \oplus \mathcal{O}_{C} \oplus \mathcal{O}_{C} \stackrel{\left(\left.\frac{\partial F}{\partial x}\right|_{C},\left.\frac{\partial F}{\partial u}\right|_{C},\left.\frac{\partial F}{\partial v}\right|_{C}\right)}{\longrightarrow} \mathcal{O}_{C}(3) .
$$

This map is $\left(0, z^{3}+\partial p / \partial u, z^{2} y+y^{3}+\partial p / \partial v\right)$, since $x^{2}$ divides $\left.p\right|_{u=0=v}$ ( $p$ vanishes scheme-theoretically on $f$ ) so that $\partial p /\left.\partial x\right|_{C}=0$. Thus the kernel is the first factor $\mathcal{O}_{C}(1)$ plus the kernel of the induced surjection $\mathcal{O}_{C} \oplus \mathcal{O}_{C} \rightarrow \mathcal{O}_{C}(3)$, which is $\mathcal{O}_{C}(-3)$ by counting degrees. 
Thus the first order deformations of $C$ are those inside the $\mathbb{P}_{1}^{2}$ over $u=0=v$, through the normal bundle $\nu_{C / \mathbb{P}_{1}^{2}}=\mathcal{O}_{C}(1)$. Writing

$$
Y_{\epsilon}=Y \times \operatorname{Spec} \mathbb{C}[\epsilon] /\left(\epsilon^{2}\right),
$$

for the thickening of any space $Y$, the deformations give thickened curves $C_{\epsilon} \hookrightarrow X_{\epsilon}$ : that corresponding to the section $s=\alpha y+\beta z \in$ $H^{0}\left(\nu_{C / \mathbb{P}_{1}^{2}}\right)$ is given by the equations

$$
u=0=v, \quad x=\epsilon(\alpha y+\beta z) .
$$

This lies inside $X_{\epsilon}$ since $x^{2}=\epsilon^{2}(\alpha y+\beta z)^{2}$ and $\epsilon^{2}=0$.

Lemma 2.3 For $p$ in (2.1) sufficiently small, and $C_{\epsilon} \hookrightarrow X_{\epsilon}$ a curve defined by $s \in H^{0}\left(\nu_{C / \mathbb{P}_{1}^{2}}\right)=H^{0}\left(\nu_{C / X}\right)$, s is not in the image of the restriction map $H^{0}\left(\nu_{C_{\epsilon} / X_{\epsilon}}\right) \rightarrow H^{0}\left(\nu_{C / X}\right)$ induced by $\left.\nu_{C_{\epsilon} / X_{\epsilon}}\right|_{C}=\nu_{C / X}$.

Remarks. Thus the curve $C_{\epsilon}$ cannot be deformed further: standard deformation theory (see e.g. [Ka]) shows that the deformation $C_{\epsilon} \hookrightarrow$ $X_{\epsilon}$ given by $s \in H^{0}\left(\nu_{C / X}\right)$ can be extended to $C \times \operatorname{Spec} \mathbb{C}[\epsilon] /\left(\epsilon^{3}\right) \hookrightarrow$ $X \times \operatorname{Spec} \mathbb{C}[\epsilon] /\left(\epsilon^{3}\right)$ if and only if $s$ is in the image of the above restriction map.

The following proof can be strengthened to show that in fact, for $s \neq \alpha(y \pm i z)$ at least, $H^{0}\left(\nu_{C_{\epsilon} / X_{\epsilon}}\right)=0$.

Proof. Repeating the above analysis of $\nu_{C / X}$ relative to the base $\operatorname{Spec} \mathbb{C}[\epsilon] /\left(\epsilon^{2}\right)$ (i.e., carrying $\epsilon$ as an extra variable, albeit one whose square is zero) shows that $\nu_{C_{\epsilon} / X_{\epsilon}}$ is the kernel of the map

$$
\mathcal{O}_{C_{\epsilon}}(1) \oplus \mathcal{O}_{C_{\epsilon}} \oplus \mathcal{O}_{C_{\epsilon}} \stackrel{\left(\left.\frac{\partial F}{\partial(x-\epsilon)}\right|_{C_{\epsilon}},\left.\left.\frac{\partial F}{\partial u}\right|_{C_{\epsilon}} \frac{\partial F}{\partial v}\right|_{C_{\epsilon}}\right)}{\longrightarrow} \mathcal{O}_{C_{\epsilon}}(3),
$$

where $s=\alpha y+\beta z$. Notice then that $\partial / \partial u$ and $\partial / \partial v$ are taken holding $x-\epsilon(\alpha y+\beta z)$, and not $x$, fixed. However $\epsilon^{2}=0$ simplifies things to make the map

$$
\left(\epsilon(\alpha y+\beta z)(2 y+q), z^{3}+\partial p / \partial u, z^{2} y+3 \epsilon(\alpha y+\beta z) y^{2}+y^{3}+\partial p / \partial v\right)
$$

with $q$ the polynomial $(\partial p / \partial x) / x$. We must show that there are no sections of the kernel restricting on $\epsilon=0$ to $s=(\alpha y+\beta z, 0,0)$, i.e., that $(\alpha y+\beta z+\epsilon l, \epsilon a, \epsilon b)$ is not in the kernel for all linear $l$ and $a, b \in \mathbb{C}$. 
Thus we must show there are no solutions in $a, b$ to

$$
2 y(\alpha y+\beta z)^{2}+a z^{3}+b y\left(y^{2}+z^{2}\right)+\left[q(\alpha y+\beta z)^{2}+a \frac{\partial p}{\partial u}+b \frac{\partial p}{\partial v}\right]=0,
$$

where the term in the square brackets can be made small with $p$. Choose $p$ such that the coefficients of $z^{3}, z^{2} y, y^{3}$ in $q, \partial p / \partial u$ and $\partial p / \partial v$ are less than $1 / 10$. Then comparing coefficients of $z^{3}$ shows that $a=0$, while the coefficients of $y\left(y^{2}+z^{2}\right)$ show that $b=0$ since $z^{2}+y^{2}$ cannot be written as a square $(\alpha y+\beta z)^{2}$.

\section{$3 \quad$ From curves to bundles}

Just as (codimension 1) divisors correspond to (rank 1) line bundles, codimension 2 subschemes $Z$ of schemes $X$ sometimes correspond to rank 2 bundles $E$ via zero sets of sections $s \in H^{0}(E)$. If this is the case the subscheme will be a local complete intersection (locally it is cut out by 2 independent equations given by the two components of $s$ in a local trivialisation of $E$ ) with normal bundle the restriction of $E$ to $Z$ (with the isomorphism set up by the derivative of $s$ at $Z$ ). Letting $L=\Lambda^{2} E$, wedging with $s$ gives maps $\mathcal{O} \rightarrow E \rightarrow L$ giving an exact sequence

$$
0 \rightarrow \mathcal{O} \stackrel{s}{\rightarrow} E \rightarrow \mathcal{I}_{Z} \otimes L \rightarrow 0,
$$

where $\mathcal{I}_{Z}$ is the ideal sheaf of functions vanishing on $Z$. We will consider $Z$ connected for simplicity.

Thus, given $Z \subset X$, for it to be the zero locus of a section $s$ of a rank 2 bundle $E, Z$ must firstly be a local complete intersection such that $\Lambda^{2} \nu_{Z / X}$ extends to a global line bundle $L$ on $X$. If this is the case then to construct $E$ we want a global extension (3.1) which is locally free (a vector bundle). Away from $Z$ this is given by an element of $H^{1}\left(L^{*}\right)$, which we may think of as an $L^{*}$-valued one-form that is $\bar{\partial}$-closed away from $Z$. Local analysis on $Z$ shows that the extension $E$ being locally free is equivalent to $\bar{\partial}$ of the form being a Dirac delta along $Z$. Thus the condition for the global existence of such a vector bundle $E$ is that the class in $H^{2}\left(L^{*}\right)$ defined by this Dirac delta is $\bar{\partial}$ of something, i.e., that it is zero. 
The upshot of all this is the Serre construction [GH], giving $(E, s)$ and an exact sequence (3.1) if and only if $[Z] \in H^{2}\left(L^{*}\right)$ is zero. Here $[Z] \in H^{2}\left(L^{*}\right)$ is defined as a linear functional on $H^{n-2}\left(L \otimes K_{X}\right)$ (the Serre dual of $H^{2}\left(L^{*}\right)$, where $n=\operatorname{dim} X$ and $K_{X}$ is the canonical bundle) by restricting a form to $Z$ and integrating:

$$
H^{n-2}\left(L \otimes K_{X}\right) \rightarrow H_{Z}^{n-2}\left(\Lambda^{2} \nu_{Z / X} \otimes K_{X}\right)=H_{Z}^{n-2}\left(K_{Z}\right) \stackrel{\int}{\rightarrow} \mathbb{C} .
$$

In particular if $H^{2}\left(L^{*}\right)=0$ then $E$ and $s$ exist.

Using this we now construct bundles $E \rightarrow X$ and $E_{\epsilon} \rightarrow X_{\epsilon}$ from $C$ and $C_{\epsilon}$. We do the $C \subset X$ case, but $C_{\epsilon} \subset X_{\epsilon}$ is identical.

$\Lambda^{2} \nu_{C / X}=\mathcal{O}_{C}(-2)$ extends to all of $X$ as $L=\mathcal{O}(-2, k)$ for any $k$; we choose $k=2$ to stand a chance of $E$ being stable. The divisor exact sequence

$$
0 \rightarrow \mathcal{O}_{P}(k-3, l-3) \rightarrow \mathcal{O}_{P}(k, l) \rightarrow \mathcal{O}_{X}(k, l) \rightarrow 0,
$$

and the cohomology of line bundles on $\mathbb{P}^{2}$ and hence $P$, show that $H^{2}\left(L^{*}\right)=0$. Thus the Serre construction gives a rank 2 bundle $E$ and a section $s$ vanishing on $C$, and so the exact sequence

$$
0 \rightarrow \mathcal{O} \stackrel{s}{\rightarrow} E \rightarrow \mathcal{I}_{C}(-2,2) \rightarrow 0 .
$$

Since the class $[C] \in H^{4}(X)$ is $\omega_{2}^{2} / 3$, the Chern classes of $E$ are $c_{1}=$ $2 \omega_{2}-2 \omega_{1}$ and $c_{2}=\omega_{2}^{2} / 3$. Thus the Chern classes of $A=E(1,-1)$ are

$$
\begin{aligned}
& c_{1}(A)=0, \\
& c_{2}(A)=\omega_{1}^{2}+\frac{4}{3} \omega_{2}^{2} .
\end{aligned}
$$

\section{An obstructed bundle}

Repeated applications of the cohomology of the sequences $0 \rightarrow \mathcal{I}_{C} \rightarrow$ $\mathcal{O}_{X} \rightarrow \mathcal{O}_{C} \rightarrow 0$ and (3.2), and Serre duality, give

$$
\begin{aligned}
H^{1}\left(\mathcal{O}_{X}(-2,2)\right)=0 \Longrightarrow H^{1}\left(\mathcal{I}_{C}(-2,2)\right) & =0 \\
\Longrightarrow & H^{1}(E)=0=H^{2}\left(E^{*}\right),
\end{aligned}
$$


and

$$
\begin{aligned}
H^{1}\left(\mathcal{O}_{X}(2,-2)\right)=0 \text { and } H^{1}\left(\mathcal{I}_{C}\right) & =0 \\
& \Longrightarrow H^{1}\left(E^{*}\right)=H^{1}(E(2,-2))=0 .
\end{aligned}
$$

Thus tensoring (3.3) with $E^{*}=E(2,-2)$ shows that

$$
H^{1}(\text { End } E) \cong H^{1}\left(E \otimes \mathcal{I}_{C}\right) .
$$

(3.3) also shows that $H^{0}(E)$ is generated by $s$, so that the restriction map $H^{0}(E) \rightarrow H^{0}\left(\left.E\right|_{C}\right)$ is zero. Thus the sequence $0 \rightarrow E \otimes \mathcal{I}_{C} \rightarrow$ $\left.E \rightarrow E\right|_{C} \rightarrow 0$ gives $H^{1}\left(E \otimes \mathcal{I}_{C}\right) \cong H^{0}\left(\left.E\right|_{C}\right) \cong H^{0}\left(\nu_{C / X}\right)$ and we find that

$$
\begin{aligned}
& H^{1}(\text { End } E) \cong H^{0}\left(\nu_{C / X}\right), \\
& H^{1}\left(\text { End } E_{\epsilon}\right) \cong H^{0}\left(\nu_{C_{\epsilon} / X_{\epsilon}}\right) .
\end{aligned}
$$

(4.1) says that $E$ has a $\mathbb{C}^{2} \cong H^{0}\left(\nu_{C / X}\right)$ of first order deformations, while (4.2) says that for any such deformation $E_{\epsilon} \rightarrow X_{\epsilon}$ given by some $s \in H^{0}\left(\nu_{C / X}\right), s$ is not in the image of the restriction map $H^{1}$ (End $\left.E_{\epsilon}\right) \rightarrow H^{1}$ (End $E$ ). This implies by standard deformation theory (e.g. [Ka]) that the component of $E$ 's moduli space (and so that of $A=E(1,-1)$ too $)$ is given by $\operatorname{Spec} \mathbb{C}[\epsilon, \eta] /\left(\epsilon^{2}, \epsilon \eta, \eta^{2}\right)$.

Thus to prove Theorem 1.1 it is now sufficient to show the slopestability of $E$ with respect to the Kähler forms $N \omega_{1}+\omega_{2}, N>1$. By replacing subsheaves of $E$ by their double duals, it is enough to show that the slopes of any line bundle subsheaves of $E$ are smaller than the slope of $E$. The cohomology of $X$ shows that the only line bundles on $X$ are of the form $\mathcal{O}(k, l)$, so it is sufficient to show that if $E(k, l)$ has a section then $\operatorname{deg} E(k, l)>0$.

The sequence (3.2) shows that $\mathcal{O}_{X}(a, b)$ has sections if and only if $a \geq 0$ and $b \geq 0$, and for a section to vanish on $C$ we must have $b \geq 1$, so that $H^{0}\left(\mathcal{I}_{C}(a, b)\right) \neq 0$ if and only if $a \geq 0, b \geq 1$. Thus we see from (3.3) that $E(k, l)$ cannot have sections unless either $k, l \geq 0$ or $k \geq 2, l \geq-1$.

So it is sufficient to show that the degrees of $E$ and $E(2,-1)$ are positive. They are

$$
c_{1}(E) \cdot\left(N \omega_{1}+\omega_{2}\right)^{2}=6\left(N^{2}-1\right)
$$


and

$$
c_{1}(E(2,-1)) \cdot\left(N \omega_{1}+\omega_{2}\right)^{2}=12(2 N+1),
$$

which are positive for $N>1$, as required.

\section{References}

[GH] P. Griffiths and J. Harris, Principles of algebraic geometry, Wiley, New York, 1978.

[K] S. Katz, On the finiteness of rational curves on the quintic 3-fold, Comp. Math., 60 (1986), 151-162.

[Ka] Y. Kawamata, Unobstructed deformations - a remark on a paper of Z. Ran, Jour. Alg. Geom., 1 (1992), 183-190.

[Mc] R. McLean, Deformations of calibrated submanifolds, Comm. Anal. Geom., 6 (1998), 705-747.

[Ty] A.N. Tyurin, Geometric quantization and mirror symmetry, Preprint, math. AG/9902027, 1999.

[Va] C. Vafa, Extending Mirror Conjecture to Calabi-Yau with Bundles, Preprint, hep-th/9804131, 1998. 